Revista Científica General José María Córdova

(Revista Colombiana de Estudios Militares y Estratégicos)

Bogotá D.C., Colombia

ISSN 1900-6586 (impreso), 2500-7645 (en línea)

Web oficial: https://www.revistacientificaesmic.com

\title{
La geopolítica chilena y su desarrollo militar (1905-2018)
}

\section{Cristian Garay Vera}

https://orcid.org/0000-0002-6575-7456

cristian.garay@usach.cl

Instituto de Estudios Avanzados, Universidad de Santiago de Chile, Chile

Citación: Garay Vera, C. (2021). La geopolítica chilena y su desarrollo militar (1905-2018). Revista Científica General José María Córdova, 19(35), 817-835. http://dx.doi.org/10.21830/19006586.807

Publicado en línea: $1 .^{\circ}$ de julio de 2021

Los artículos publicados por la Revista Científica General José María Córdova son de acceso abierto bajo una licencia Creative Commons: Atribución - No Comercial - Sin Derivados.

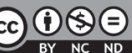

Para enviar un artículo:

https://www.revistacientificaesmic.com/index.php/esmic/about/submissions
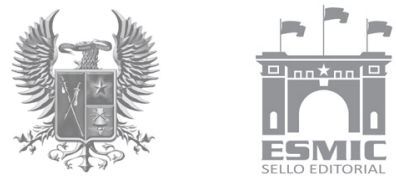


\title{
La geopolítica chilena y su desarrollo militar (1905-2018)
}

\section{Chilean geopolitics and its military development (1905-2018)}

\section{Cristian Garay Vera}

Instituto de Estudios Avanzados, Universidad de Santiago de Chile, Chile

\begin{abstract}
Resumen. Este artículo postula la importancia de la geopolítica militar como columna central del pensamiento geopolítico chileno. Para el autor, esto es producto tanto del énfasis por asesorar y ejecutar directrices en este campo como de la reflexión teórica desde principios del XX en el seno del Ejército y la Armada de Chile. Desde esta perspectiva, el cultivo de la geopolítica se ha ligado a nociones de estrategia, geografía política y planificación militar. El artículo sugiere cuatro fases de su desarrollo que dependen del contexto político del país, como también de su mayor o menor acceso a la toma de decisiones del Estado. Como resultado, se establece que el contexto político del país, la autonomía militar o el control civil de las Fuerzas Armadas, entre otros factores, han influido en el desarrollo de la geopolítica en Chile.
\end{abstract}

Palabras ClaVE: Chile; doctrina militar; Fuerzas Armadas; geopolítica; soberanía; territorio

ABSTRACT. This article postulates the importance of military geopolitics as the central pillar of Chilean geopolitical thought. For the author, this is the product of both the emphasis on advising and executing guidelines in this field and the theoretical reflection within the Chilean Army and Navy since the beginning of the 20th Century. From this perspective, the development of geopolitics has been linked to notions of strategy, political geography, and military planning. The article suggests four phases of development that depend on the country's political context and the state's greater or lesser access to decision-making. As a result, it is established that the national political context, the military autonomy, or the civil control of the Armed Forced, among other factors, have influenced the development of geopolitics in Chile.

KEYwORDs: Armed Forces; Chile; geopolitics; military doctrine; sovereignty; territory

Sección: Dosier • Artículo de investigación científica y tecnológica

Recibido: 3 de febrero de 2021 • Aceptado: 30 de mayo de 2021

ConTACTO: Cristian Garay Vera cristian.garay@usach.cl 


\section{Introducción}

Sin duda, la geopolítica ha constituido una ciencia militar por excelencia. Fue particularmente relevante en los tiempos de la Guerra Fría, pero, en un contexto mucho más amplio, se desarrolló como una forma de pensar el Estado centrada en sus intereses estatales. Desde el nacimiento de la geopolítica clásica hasta 1990, esta forma de pensar el Estado pretendió abarcar un horizonte cognitivo integral; pero desde entonces la geopolítica ha cambiado de enfoque y se ha pluralizado, como lo advierte un estudio reciente (Mendoza, 2018). En este sentido, este artículo parte del reconocimiento que hace Howard T. Pittman (1990, p. 177) de la existencia de una escuela geopolítica chilena y de una integración entre el gobernante y el geopolítico en Augusto Pinochet Ugarte, aunque sin ignorar la posibilidad de que esa relación subordine la geopolítica a este liderazgo e impida una mirada más integral del desarrollo geopolítico militar hasta la actualidad.

El propósito de este artículo es describir el desarrollo institucional de la geopolítica chilena en el ámbito docente y analítico desde el siglo XX hasta el año 2018. Aunque la geopolítica no es per se un saber militar, fue el Ejército y luego la Armada de Chile los que primero se apropiaron de esta disciplina, en un momento que se desarrollaba paralelamente a la geografía. La hipótesis es que este desarrollo tuvo que ver con la necesidad de un enfoque complementario a lo estrictamente geográfico que influyera sobre los tomadores de decisión civiles y que incluyera nociones como desarrollo, soberanía y participación de las Fuerzas Armadas.

Para ello se usa el método genealógico, mediante el cual se trazan los orígenes y los préstamos intelectuales tanto foráneos como propios en la formulación geopolítica chilena, que derivan del cultivo endogámico de esta rama en la profesión militar. Luego se distinguen las diferencias en sus planteamientos entre la geopolítica terrestre y la naval. Con este fin, se identifican los autores militares relevantes, sus influencias, textos y repercusiones en la doctrina de la institución y, eventualmente, en el discurso de política exterior. En este sentido, se acoge la tesis de que la profesión militar vio en la geopolítica un nicho propio, y así la constituyó en ciencia o saber militar (Mendoza, 2018, p. 18). Con posterioridad, la bibliografía crítica de los regímenes militares vio en la geopolítica una ciencia geográfica y de aplicación militar, ligada a una perspectiva profesional que entiende las relaciones internacionales y los conflictos primordialmente como manifestaciones estatocéntricas, bajo un parámetro estrictamente realista.

Por otro lado, la geopolítica ha tenido rasgos comunes en América Latina, por lo cual son numerosos los autores de origen militar: "Jorge Atencio, E. Backhauser, A. Pinochet, J. von Chrismar, Golbery do Couto e Silva y Alberto Escalona Bravo, por mencionar algunos", cada uno de los cuales subraya la idea de que la geopolítica es "un importante conocimiento de carácter fundamental para estadistas y estrategas" (Mendoza, 2018). Más recientemente, la geopolítica naval introdujo un concepto más allá de lo interestatal 
al considerar tanto los aspectos de soberanía económica nacional como la realidad de una alta mar con un régimen de gobernanza.

Con este método de relacionar los autores, textos y planteamientos con el desarrollo disciplinar de la geopolítica en un contexto de educación militar, se puede entender que esta disciplina trató de modernizar y apropiarse de los desarrollos teóricos provenientes de una valoración política de la geografía. Esto la distingue de otra rama en la formación educativa castrense que es la geografía militar, destinada a dar conocimientos específicos acerca del uso militar de la geografía en las operaciones y que no tiene la misma trascendencia en la asesoría al mando.

En este sentido, se considera que la constitución de la geopolítica chilena ha estado motivada por la consideración de la insularidad de Chile en su región y ante sus vecinos, dadas las dificultades físicas de comunicarse con el resto salvo por mar o atravesando las alturas de la cordillera de los Andes. $\mathrm{Al}$ respecto, se debe tener en cuenta especialmente el pensamiento del prócer de la Independencia Bernardo O'Higgins (1778-1842) acerca del destino magallánico (Manzano, 2018), así como el pensamiento posterior del ministro Diego Portales Palazuelos (1793-1837). Ambos autores serían geopolíticos prácticos a la usanza británica. Respecto a O'Higgins, Manzano (2018) hace hincapié en que en su formación británica tuvo predilección por los asuntos navales: "Con ello, se forjó la idea de la relevancia que debe tener el poder naval de un país para sellar su destino, algo que se ve reflejado en sus cartas" (p. 29).

A partir de la pregunta de investigación sobre qué aspectos del cultivo de la geopolítica están asociados al desarrollo teórico y analítico de las Fuerzas Armadas chilenas, este trabajo describe la evolución de la escuela geopolítica militar chilena, con base en las variables de texto/contexto, representantes, temas, temática terrestre y/o marítima, relación con el pensamiento civil, y control civil/autonomía militar. Para efectos de la presentación, se establecen cuatro periodos de la historia chilena que dan cuenta de la evolución que han tenido las Fuerzas Armadas en su participación directa e indirecta con el Estado y la toma de decisiones.

\section{El momento "clásico": los orígenes (1905-1948)}

Lo característico del momento clásico del origen de la geopolítica es que obedece a un desarrollo europeo de la ciencia social, en el cual estaba en su cénit la impronta spenceriana y darwinista, y que tuvo en Friedrich Ratzel como su fundador un eje de convergencia con la geografía, ya que fundó al mismo tiempo la geopolítica y la geografía como disciplinas (Cairo, 2011, p. 338; Paulsen, 2015). Bajo ese contexto fundacional, se racionalizó la idea de que el comportamiento es influido por la posición geográfica, idea que evolucionaría hacia un determinismo de las fronteras asociado al crecimiento del Estado nacional. Por tanto, reconocer las leyes de este desarrollo se consideraba esencial para la evaluación del entorno territorial de un Estado y su proyección internacional. Así, el enfoque geopolítico 
se desarrolló al amparo de la cátedra de Geografía en la Academia de Guerra del Ejército, lo que determinó los planteamientos que conducirían a la geopolítica.

En todo caso, el desarrollo de la geografía y de la geopolítica no son militares. Corresponde a un momento de profesionalización de una ciencia sobre el espacio y sus características. Ambas ramas, que nacieron juntas, tenían un protodesarrollo en la cartografía, las teorías de la influencia del clima y el terreno sobre las sociedades, y las fisonomías de pueblos y comunidades en el globo. Pero particularmente en América del Sur, además del interés científico por estas disciplinas, hubo un interés profesional de los militares en estas disciplinas como elementos auxiliares de la comprensión estratégica, de lo cual fueron evidencia Chile, Argentina y Brasil. Pero incluso en estos hubo también cultores civiles de la talla del Barón de Río Branco en Brasil o Estanislao Zeballos en Argentina.

En Chile, el interés por la geopolítica vino mezclado con el de la geografía. Si la geografía se correspondía con la táctica, la geopolítica, con su pretensión de formular leyes acerca del desarrollo de los Estados, se aproximaba a la estrategia y al alto mando. En un Estado con un control civil de los militares, la oficialidad fue asimilando los conceptos de formas científicas de la guerra también en el cultivo de las ciencias geográficas. De ello resultó que uno de los generales más destacados de la etapa de la "prusianización" del Ejército, Jorge Boonen Rivera, escribió en $1905^{1}$ su obra Ensayo sobre la geografía militar de Chile (1905). Esta obra, necesaria para la planificación de las campañas terrestres, fue acompañando la introducción de la geopolítica clásica. En 1923 se difundieron las obras de Haushofer, Kjellen y Ratzel en el Ejército. La presencia de estos autores se puede comprobar en el catálogo de la biblioteca de Cañas Montalva, donde figuran textos de geopolíticos tempranos: nada menos que "nueve obras de Kjellen, 7 de Haushofer, 3 de Ratzel y 3 de Weigert" (Garay, 2008, p. 22). Santis (1988), por su parte, documenta la lectura e influencia de Ratzel en la geopolítica chilena. Este interés se expresa, además, en que en la Biblioteca del Congreso de Chile figura el libro de Karl Haushofer Geopolitik des Pazisfishen Ozeans: Studien über die Wechselbeziehungen Zwischen Geographie und Geschichte, publicado en Berlín en 1938. Aparte de este título, hay 449 ingresos referidos a geopolítica en el catálogo.

Los autores militares reconocen el año de 1943 como el nacimiento oficial de esta perspectiva en la educación militar. Para Ortega (2014), la tríada inicial de la geopolítica fue formada desde 1944 por "Ramón Cañas Montalva, Humberto Medina Parker y Romeo Barrientos Rosas" (p. 126). Se toma ese año porque el primer artículo de geopolítica fue de 1943, pero sobre todo por la publicación de la traducción en el

1 Jorge Boonen Rivera (1858-1921) hizo la evaluación de las academias militares de Espańa y Alemania en 1884. Más tarde fue Inspector General del Ejército por 11 ańos desde 1910. Políglota, asentó la influencia alemana. Para Ortega (2010), su influencia como geógrafo se refleja en los generales "Pedro Charpin Vidal, Indalicio Téllez, Guillermo Barrios Tirado, Ramón Cañas Montalva, Bernardino Parada Moreno y Augusto Pinochet Ugarte, entre otros" (p. 35). 
Memorial del Ejército de Chile del texto "Geopolítica. La fantástica carrera de un sistema científico que un británico inventó, los alemanes usaron y los americanos necesitan estudiar" de Joseph J. Thomike Jr. En 1944 aparecieron cuatro artículos en diferentes números del Memorial del Ejército de Chile del Teniente Coronel Humberto Medina Parker que difunden el pensamiento de los fundadores de la geopolítica: "La geografía y la geopolítica", "Las leyes para el crecimiento espacial de los Estados", "Rudolf Kjéllen y sus perspectivas geopolíticas" y "El mago de la geopolítica" (García, 2005, p. 10). Luego, en 1948, Medina Parker publicó su conferencia Sangre y suelo de Chile. La geopolitica en acción, que reprodujo Ortega en 2014, en la que reitera y sintetiza sus puntos de vista anteriores.

Por su parte, Cañas Montalva empieza a escribir un año después, en 1945, y alcanza su cénit en 1948. Identifica como elemento central la tesis de la Era del Pacífico (Haushofer) y la conecta con la situación de control de Chile en sus dimensiones marítimas de control de los pasos australes y la Antártida. Una característica del pensamiento geopolítico de Cañas Montalva es su tridimensionalidad, que abarca lo terrestre, lo marítimo y lo aéreo, porque sitúa a Chile en la confluencia de las rutas de conexión mundiales. Él mismo habla de una "edad del aire" (Cañas, 1959, p. 4). En lo marítimo, Cañas Montalva postulaba que el paso entre el Atlántico y el Pacífico es equivalente, geopolíticamente, al del canal de Panamá. Y en este caso, a Chile le correspondía el control pleno y soberano en la punta sur del continente, que se proyecta con la cordillera que da origen a la Antártida (Garay, 2008). Así, Cañas se centraba en la afirmación y el establecimiento de soberanía antártica y de bases polares. Su mayor influencia se reflejó en la interacción con los mandos políticos y militares respecto de la soberanía en el arco Pacífico-Antártico, así como en su relación privilegiada con tres presidentes radicales de la época en los asuntos de la Antártida chilena y sus conflictos con Argentina y Reino Unido.

La geopolítica de Cañas, Barrientos y Medina surge en esta época en un contexto democrático de control civil y en la adhesión de Chile al bloque democrático durante la Segunda Guerra Mundial. Sus planteamientos, como se ha visto, reflejan los postulados iniciales de la geopolítica a nivel mundial con adaptaciones al entorno del Estado chileno, como el desarrollo de una concepción de Chile como país tricontinental y poseedor de "la llave" del Pacífico Sur.

\section{La Guerra Fría, el alineamiento y el enfoque vecinal (1949-1973)}

Luego de su momento de origen, la geopolítica clásica se actualizó combinándose con conceptos de las relaciones internacionales afines al ambiente de la Guerra Fría, tales como la contención, la distensión y el equilibrio bipolar. Se mantuvo la tesis del aislamiento de Chile y su condición de "isla", haciendo contrapunto entre su alineamiento hemisférico y global, y las necesidades de seguridad en el entorno vecinal. Aunque se seguían citando los 
maestros clásicos, entraban en juego autores estadounidenses como Spykman en el marco de alineación ${ }^{2}$.

Las tesis fundamentales de Cañas Montalva se expusieron entre 1945 y 1948. A ellas sucedieron más tardíamente en el Ejército las ideas de Augusto Pinochet, que escribe Geografía militar. Interpretación militar de los factores geográficos (1967), y luego, recién ascendido a Coronel, publica en el Memorial su trabajo "Geopolítica. Diferentes etapas para el estudio geopolítico de los Estados" (Pinochet, 1968). El pensamiento de Pinochet es deudor, como lo demuestra Peña (2013), de los cursos del Coronel Rodríguez Tascón (1950a; 1950b). Si bien hay transliteraciones de autores alemanes, también es cierto que habitualmente cita al comienzo de sus obras estas fuentes. Por otra parte, la influencia de Cañas Montalva reapareció con su discípulo, el Coronel Julio von Chrismar Escuti, autor de Leyes que se deducen del estudio de la expansión de los Estados (1968). En cambio, el pensamiento del Coronel Medina Parker salió de esta primera línea de promotores de la disciplina en los siguientes años.

Lo más relevante de la actividad del Teniente Coronel Pinochet fueron sus clases en la Academia de Guerra; más que su desarrollo teórico, fue importante su condición de difusor de la geopolítica (Garay, 2006, p. 151). En 1955, Pinochet cambió el nombre del curso de Geografía Militar a Geografía Militar y Geopolítica. Antes había escrito un texto de geografía militar: Sintesis geográfica de Chile Argentina, Bolivia y Perú (Pinochet, 1953). En esos años, como profesor de la Academia de Guerra de Ecuador, Pinochet redactó los programas de varias asignaturas, entre ellas Geopolítica (1956-1957) (Pinochet, 1990).

Aunque, como se ha dicho, se sigue cuestionando la originalidad de sus textos, es indudable que la geopolítica tenía una presencia relevante en su discurso, que luego influiría desde el poder a partir de 1973 (Amorós, 2019). El tono de sus obras es de reflexión sobre la situación geográfica de Chile en el entorno de la seguridad regional y nacional. La influencia de Cañas Montalva, si bien no es citado por Pinochet, reapareció desde la crisis con Argentina, a partir de 1978, en los escritos militares (Caviedes, 1990, pp. 18-19).

En esta época apareció una primera geopolítica marítima con repercusión en el concepto de Zona Económica Exclusiva y Patrimonial, basada en el nacionalismo económico del Partido Radical, que estuvo en su cénit durante tres presidencias de 1938 a 1952. Esta geopolítica se refleja en la doctrina de las 200 millas marítimas (1947) formulada por Gabriel González Videla (Witker en Devés \& Álvarez, 2020), que recibió el apoyo de la Armada y se concretó en ese momento en la idea de bloque del Pacífico. En años posteriores, en la Revista de Marina, con autores como Hernán Cubillos L. (1950) y Arturo Troncoso D. (1961), se publicaron textos que

demostraron que las democracias occidentales alcanzaron mayor desarrollo intelectual y sentido de la realidad respecto del resguardo por parte de Chile en la utilización del

2 Nicholas Spykman (1893-1943). Basado en Mahan, pero sobre todo en Mackinder, sostuvo la importancia del rimland en la hegemonía mundial. Influyó en Kennan, Kissinger y Brzezinsky. 
estrecho de Magallanes, el canal de Beagle y el mar de Drake, para el caso de guerra mundial o conflicto con la Argentina. (Caviedes, 1990, p. 19)

Ese mismo razonamiento explica la convergencia del alineamiento con Estados Unidos y la contención de las ambiciones bioceánicas argentinas (Garay, 2017). En la apreciación geopolítica al uso en ese momento, Chile era visualizado como un apoyo al esfuerzo estadounidense. La idea de construir en 1945 un aeropuerto para el aterrizaje de grandes transportes aéreos en Punta Arenas tenía por objeto posibilitar ayuda estadounidense para defender el Estrecho de Magallanes de los soviéticos (Garay, 2017, p. 189). Esto se dio en una perspectiva geopolítica nacional inserta en la situación mundial de una posible tercera guerra mundial, en la cual se planearon ampliaciones de los aeropuertos militares en Antofagasta, Santiago, Puerto Montt y Punta Arenas que sirvieran tanto para la defensa nacional como para los planes hemisféricos de defensa.

\section{“Ciencia del Estado” (1973-1990)}

Como conclusión podemos decir que la geopolítica, con sus estudios, determina los objetivos políticos que debe alcanzar el Estado, y aconseja al conductor cual sería el procedimiento más eficaz para obtenerlo.

Pinochet (1984, pp. 37-38)

El ascenso de las Fuerzas Armadas al poder bajo el liderazgo de Augusto Pinochet implicó una revalorización de la geopolítica. A partir de entonces, esta se vertió sobre asuntos de seguridad nacional e internacional, abarcando desde aspectos de las relaciones internacionales hasta la política administrativa del país en el nuevo contexto refundacional. Se convirtió entonces en conocimiento obligatorio para los oficiales destinados a cargos de responsabilidad administrativa en el Ministerio de Relaciones Exteriores y otros ministerios. Adquirió el carácter de una "ciencia del Estado", bajo la cual se toman decisiones de carácter territorial y se hacen planificaciones de desarrollo. Así se comenzó a aplicar al análisis demográfico, las migraciones y la soberanía nacional, prestando especial atención a zonas extremas donde hay conflictos territoriales. Este análisis influyó en la creación en 1974 de la Comisión Nacional para la Reforma Administrativa (Conara), para diseñar una nueva división administrativa que creaba 12 regiones en vez de 25 provincias; la creación de la Zona Franca de Iquique (ZOFRI) en 1975, y la construcción de la Carretera Austral en 1976.

En este periodo, los funcionarios militares tienden a considerar la geopolítica como una herramienta científica, de carácter auxiliar para el mando político militar según corresponda. Eso repercutió en la formación de la Academia de Guerra al aumentarse el número de horas dedicadas al ramo de geopolítica en los Cursos Regulares de Estado 
Mayor (CREM I, II y III): 64 (1960-1973); 90 (1975-1977); 112 (1977-1981), 108 (1981-1988) y 80 (1989) (García, 2005). En la Armada, la geopolítica mantuvo su perfil inserto en los ramos, pero no tuvo este marcado incremento horario.

Desde el Ejército de Chile se difundió la geopolítica y constituyó una instancia del Ministerio de Defensa Nacional para dictar cursos a todas las ramas de las Fuerzas Armadas, y en 1975 se creó la Academia de Seguridad Nacional (actual Academia de Estudios Estratégicos y Políticos, ANEPE). Para ampliar el cultivo de la disciplina, se creó el Instituto Geopolítico de Chile, fundado en 1981 con apoyo del Ministerio de Defensa, que publicó la Revista Chilena de Geopolítica, y donde predominaban especialistas civiles: los geógrafos de la Universidad Católica Hernán Santis (1988), Mónica Gangas (Gangas \& Santis, 1987) y Ricardo Riesco (1985), y Mario Arnello (1985). Riesco fue nombrado posteriormente embajador en Alemania durante el mando de Pinochet. Por su parte, Mario Arnello fue diputado del Partido Nacional, embajador ante la ONU y la OEA (1974-1978) y director de la Dirección de Bibliotecas, Archivos y Museos (1986-1990). Este núcleo académico expandió el pensamiento geopolítico en sectores académicos y administrativos.

Los geopolíticos militares más relevantes de este periodo, siguiendo las entrevistas realizadas y los registros de obras, son Augusto Pinochet, Germán García Arriagada (1989), Jaime García Covarrubias (1985) y Julio von Chrismar ${ }^{3}$, en orden de importancia. La obra central de este periodo es la de Augusto Pinochet, Geopolítica, publicada originalmente en 1974 (1984), que además fue traducida. En estas obras se sigue la matriz clásica. Pinochet (1984) cita dos autores, Kjellen y Haushofer, y da su propia definición:

La Geopolítica es una de las ramas de las ciencias políticas que, basada en los conocimientos geográficos, históricos, sociológicos, económicos, estratégicos y políticos, pasados y presentes, estudia en conjunto la vida y desarrollo de una masa humana organizada en un espacio terrestre, analizando sus múltiples y recíprocas influencias (sangre-suelo) para deducir sus objetivos y estudiar sus proyecciones, con el fin de lograr en el futuro un mayor bienestar y felicidad para el pueblo.

Reiteraba esta idea diciendo que la geopolítica

Ha dejado de ser solo una ciencia agresiva entre los Estados para convertirse en una sana consejera del conductor, a quien, científicamente, le señala los fines del Estado y cuál sería la forma como podría alcanzarlos en el futuro, para brindar con ello, paz, dicha y bienestar a su pueblo. (Pinochet, 1984, p. 23)

En un punto de su reflexión decía:

La Geopolítica no es rama de la ciencia geográfica sino de la ciencia política: Indaga los movimientos que acarrean cambios, alteraciones y transformaciones de situaciones

3 En ese periodo, Julio von Chrismar no publicó obras, pero ejerció la docencia e hizo asesoría. 
existentes y estudia los resultados que de aquellos derivan. Ella indaga la dinámica de los espacios terrestres, por lo que algunos geopolíticos deducen que la Geopolítica no puede ser una ciencia general ya que cada nación reacciona de manera distinta ante los factores geográficos y sociopolíticos del Estado. La localización, el espacio, el suelo, el clima, el mar, el conglomerado humano y otros factores, tienen un significado diferente para cada Estado, por cuanto no existen naciones geográficas que sean iguales, ni aun análogas. Luego debe existir una Geopolítica general y una particular para cada país o Estado. (Pinochet, 1984, p. 37)

A diferencia de los autores de la época clásica, Pinochet concibe la geopolítica dentro de la ciencia política y no cercana a la geografía. Entiende, de modo práctico, que su fin es aconsejar al poder, guiar y planificar, más que elaborar diagnósticos acerca del Estado. Pero en este desarrollo teórico hay una deriva práctica que se traduce en discusiones de políticas futuras. En sus aplicaciones, los cultores militares diagnosticaban los problemas territoriales y demográficos de Chile. Sostenían que hay un "núcleo vital”, opuesto a los débiles extremos del territorio, basando esta distinción en la baja densidad de población, e identificaban la falta de mecanismos de incentivos para las zonas extremas. Por tanto, en los años ochenta, el gobierno de Pinochet incentivó iniciativas de colonización y dio facilidades para las actividades económicas en esas zonas (García, 1989; 2000; García, 1985). Al respecto, algunos oficiales tuvieron responsabilidades en el diseño de la Carretera Austral, como el General Julio Canessa Robert (1982) y el Coronel Germán García Arriagada (1989 y 2000). Como dice uno de los autores considerados:

[...] la definición de "Núcleo Vital" —explica uno de ellos—, con la cual concuerdan los entendidos en la materia, afirma que es la zona de mayor densidad de población (sic), por lo tanto, es unívoco con "nuclear", siendo lógico que en esta zona se desarrollen las principales vías de comunicaciones y se instalen los poderes directivos del Estado. (García, 1984, p. 84)

Como se evidencia, la geopolítica y los desarrollos territoriales fueron impulsados por Pinochet en este periodo. Así surgió la Zona Franca de Iquique (reactivada por Pinochet en 1975) y su similar de Punta Arenas (1977), regidas con el Decreto con Fuerza de Ley (DFL) n.o 341 de 1977, así como la Ley Navarino de 1985 para el fomento de las actividades económicas en Tierra del Fuego (Medalla, 2008). Se enfatizó la conectividad al extremo sur mediante la Carretera Austral, obra iniciada por el Cuerpo Militar del Trabajo en 1976 y cuya planeación algunos anteceden en estudios del propio Pinochet de 1956 (Schiappacasse et al., 2012, p. 476; Medalla, 2008). En 1982 se inició la segunda fase, la Red Básica Austral, que terminaría de construirse en 1988. En los veranos, el General Pinochet solía visitar la obra. Desde luego, el razonamiento geopolítico ya no estaba radicado en el nivel militar, sino en el político; en ese contexto hay funcionarios civiles y académicos que reclaman una visión geopolítica de largo plazo. Gonzalo Rojas (2000) transcribe una cita muy ilustrativa, pues, en palabras del General Pinochet, se trata de 
[...] poner término al aislamiento de una extensa zona en la parte sur y austral del país, [lo que] tiene un significado geopolítico y de desarrollo económico de gran trascendencia nacional: Así vamos a unir toda la zona, con la gran visión y sentido geopolítico de integración que tiene esta Carretera Austral. (p. 605)

En el contexto de varios problemas fronterizos con Bolivia, Perú $(1974,1978)$ y Argentina (1978 y 1982), se buscaba reducir la vulnerabilidad producida porque el territorio continental chileno era discontinuo y había que utilizar vías terrestres argentinas para llegar a Magallanes y Aysen. Este aspecto no pasó desapercibido; Pinochet lo recalcaba y por eso manifestó: "insistimos que las vías de comunicaciones tienen una influencia decisiva en la unificación de las naciones" (citado en Medalla, 2008, p. 86).

Eso era en el plano nacional y vecinal. Otra vertiente de este análisis geopolítico era la asimilación de la tesis de la Era del Pacífico. Para mitigar el bloqueo occidental, y también por razones económicas (vender el cobre a Japón y China), Pinochet alienta el despliegue de embajadas sobre Asia Pacífico. En una visión que data del año 1972, cuando calificó como la noticia más relevante del ańo la visita de Nixon a la China Popular, Pinochet tomó por proféticas las alusiones de Haushofer sobre la importancia del Pacífico en las relaciones internacionales y del mundo asiático. Chateau (1977) hizo constar que los planteamientos geopolíticos del régimen militar habían trascendido a la transición democrática.

En esta tercera fase aparecen autores con incidencia sobre la temática marítima que buscaban formular enfoques tendientes a la democratización y cooperación con el área del Pacífico Sur. A fines del periodo aparece Chile geopolitico. Presente y futuro, del Almirante Óscar Buzeta (1978), bajo un contexto de convergencia reminiscente con orientaciones integracionistas (Pacto Andino, ALALC) y de simpatías demócrata-cristianas, lo que significó su distanciamiento de actividades docentes en la Armada ${ }^{4}$. Frente a este enfoque, la línea dominante fue la del Almirante Francisco Ghisolfo (1979; 1982), que se concentró tanto en la estructuración de un "poder marítimo" como en los requerimientos operacionales de la Armada frente al espacio marítimo y la disputa con Argentina. A juicio de los profesores Carvajal y Monteverde (2012):

El almirante Ghisolfo concentra su análisis geopolítico y geoestratégico en la estructura insular de Chile, especialmente en la importancia geográfica de la Isla de Pascua. La Geopolítica de Ghisolfo es una geopolítica militar, orientada a la formulación de una estrategia naval. (p. 53)

La Revista de Marina fue el núcleo de estas posiciones más ligadas a la Armada de Chile. Así, el Capitán Carlos di Giorgo (1979) sustentaba la necesidad de crear una Subsecretaría de Marina Mercante y un Instituto del Mar, que congregara los esfuerzos

4 Buzeta escribió que el concepto integracionista de E. Frei, entonces en la oposición, estaba basado "en la realidad geográfica de Chile y de los países andinos y en una fundamentada previsión geopolítica" ("Confidencias", 1979). 
por constituir un poder marítimo que abarcara la marina de guerra, la marina mercante, los intereses marítimos, el subsuelo marino y la industria naval. A diferencia de la geopolítica del Ejército, la Armada buscaba satisfacer necesidades de despliegue y "materializar una geopolítica oceánica”. Como decía el Capitán de Navío Carlos Perey (1986), había que compatibilizar la custodia de los espacios marítimos y la fuerza militar con la necesidad de crear una conciencia marítima para aprovechar la explotación del mar.

\section{Diversidad y adaptación en el contexto de redemocratización (1990-2018)}

Dado el cambio político de la redemocratización, otras áreas (políticas públicas, por ejemplo) pasan a ocupar el lugar de la geopolítica. Aunque sus cultores abandonan sus aspiraciones de coordinar las políticas estatales, la geopolítica conserva su enfoque y diagnóstico. Así, la disciplina retorna al ámbito militar y debe conciliar la gradual desaparición de la hipótesis de un conflicto con Argentina — al menos de modo formal— con la integración con ese país en instancias como Mercosur. Asimismo, el giro brasileño a raíz de la convergencia en el Mercosur obliga a desligar la idea del contrapeso entre Brasil y Argentina. Esto tendrá consecuencias sobre el planteamiento de la geopolítica chilena que serán características de la fase transicional. La primera es la búsqueda de asimilar la disciplina a enfoques más cercanos a la cooperación, la integración y el desarrollo que al conflicto; también se problematiza conceptualmente su papel en el ambiente de redemocratización.

Germán García Arriagada aborda estos cambios en un enfoque retrospectivo y los expone a la institución ${ }^{5}$ en “¿Quo Vadis Geopolítica?” (2005). El trabajo de ese autor es interesante, ya que revisa la situación de la disciplina en el mundo y somete a un cuestionario a profesores especializados de fuera del país —especialmente J. Child et al., autores de Rethinking geopolitics (1998)—, integrando o asimilando conceptos y enfoques más contemporáneos, como la corriente crítica. Igualmente destaca el hecho de que el Estado perdía su condición de actor central y unitario del sistema internacional con la globalización a partir de los noventa, lo que suponía la renovación del marco rígido estatocéntrico (García, 2005, p. 6). También dirige su atención críticamente a los corredores bioceánicos y a formas de articulación regional determinadas por el Mercosur y otras instancias regionales que "abrían" geopolíticamente a Chile con Argentina. El artículo de García Arriagada (2005) es una revisión acerca de los nuevos aires de la disciplina, útil también para la actividad en sus cursos como docente de la Academia de Guerra, determinando sus orientaciones al futuro. En suma, resulta receptivo de la globalización y de los cambios políticos al interior del país.

$5 \quad \mathrm{Al}$ ser publicado en el Memorial del Ejército, su trabajo quedó comprometido por la Dirección de la Academia de Guerra para ser difundido en el Anuario 2005-2005 de la Academia de Guerra del Ejército de Chile. 
Paralelamente, la geopolítica marítima se profundiza con Jorge Martínez Bush, Comandante en Jefe de la Armada y autor de Oceanopolitica (1993), que centra su preocupación en la gestión de recursos marítimos, la delimitación marítima de las zonas económicas exclusivas y empieza a perfilar posiciones respecto del rol de Chile en el Pacífico y los temas de gobernanza de alta mar (Duvauchelle, 1996), que también se tratan en ambientes jurídicos y diplomáticos.

Un enfoque más diversificado de la geopolítica se planteó para el concepto de fronteras interiores y más tarde el de Chile-País Puerto. En el primer caso, se hizo un análisis crítico acerca de la soberanía efectiva y el poblamiento al interior de Chile, mientras el otro destacaba el valor de la posición geográfica en relación con el intercambio entre Asia y América del Sur. También en el escenario de estos cambios se hizo mención del problema demográfico y de tasa de natalidad: el Coronel César Strait (1998) respecto a Aysén, y el General Gonzalo Santelices (1998) respecto de la población y poblamiento de Chile.

El 25 de octubre de 1994 se creó la Comisión Nacional para el Estudio e Incorporación de las Fronteras Interiores al Desarrollo Nacional (1994), fruto de un trabajo de una comisión del Ejército de Chile. Esa comisión elaboró el Mapa de las Fronteras Interiores del país, que representaba las dificultades de acceso, servicios y baja población, considerando las zonas con menor presencia estatal y soberanía. Este mapa permitió agregar servicios públicos y diseñar políticas públicas. Aunque algunas de las propuestas no se desarrollaron más, los enfoques que convergen en las fronteras interiores se mantienen como tema local. Así, por ejemplo, Ortega (2009):

La forma alargada de Chile, unido a su desmembramiento en el sur y las continuas sectorizaciones que imponen sus ríos y quebradas transversales, tienen un efecto adverso para el desarrollo y para la acción beneficiosa del Estado. Las decisiones tomadas para disminuir las fronteras interiores y para la conectividad de todo el territorio son un esfuerzo inacabado. (p. 65)

No obstante, en una obra posterior, Ortega (2015) manifestó más bien cierto escepticismo respecto a la concreción de los postulados discursivos. En efecto, el periodo en cuestión, 1990-2018, se caracteriza por la ausencia de influencia de planteamientos geopolíticos, salvo en el tema de las fronteras interiores. En la institución, la agenda investigativa se prolonga en trabajos de Pedro Orueta (1999) y Carlos Meirelles (2000), y especialmente en la Academia de Guerra del Ejército por el profesor Coronel Rodolfo Ortega $(2008 ; 2009 ; 2010 ; 2014 ; 2015)$.

Los aires de renovación disciplinar se expresaron en las Primeras Jornadas Académicas Internacionales de Geopolítica en la Academia de Guerra en 1997. Aunque todavía estaba la presencia del comandante en jefe Pinochet, que dictaba su clase magistral y entregaba su libro La Carretera Austral, su impacto y proyección, el debate subsiguiente lo planteó el comandante de institutos militares Juan Emilio Cheyre, quien terció sobre su vigencia: 
La Geopolítica se presenta hoy como una herramienta del Estado para hacer frente a los cambios de un nuevo esquema internacional en que las fronteras no necesitan ser "movidas" para aspirar a mayores niveles de progreso y seguridad, porque estas están siendo cada vez más difusas y permeables. (citado en Araya, 1997, p. D26)

En este enfoque, la geopolítica, decía Cheyre, debe hacerse de manera más amplia, para hacerse cargo de las nuevas amenazas "dentro de las cuales la [dimensión] militar es solo una de ellas y quizás no la más prominente" (Araya, 1997).

Para Pinochet, por su parte, el escenario mundial se caracterizaba por el desequilibrio en el espacio estratégico; por la diversidad y complejidad de los mercados, y la deuda y el déficit, en la dimensión económica; y por la inestabilidad en el campo de la seguridad. Precisó que Chile era parte del "mundo americano" con "una creciente presencia en el mundo asiático-Pacífico" y con fuertes lazos culturales, raciales, culturales y emocionales con Europa (citado en Araya, 1997). Decía que el futuro sería de cuatro o cinco potencias fuertes, sin un líder claro que articulara el sistema internacional.

Respecto a esto, Ortega (2010) describió las tribulaciones de la tradición geopolítica chilena. Al consultar al comandante en jefe del Ejército, Juan Emilio Cheyre escribió:

En la actualidad, como se nos presenta en el libro, la Geopolítica se ha revitalizado. Participa en el estudio de los problemas globales, como la lucha por la energía, las migraciones humanas, el acceso al agua, la alimentación, el narcotráfico y otros desafíos que la humanidad enfrenta como un todo. (citado en Ortega, 2010, p. 10)

Cheyre (1997), en una reflexión marcada por la contemporaneidad, extiende el análisis al contexto de la ecología, los recursos y la preservación del ambiente físico. En el marco de las citadas Primeras Jornadas Internacionales de Geopolítica, Cheyre sostuvo que la diferencia entre la geografía política y la geopolítica era que la primera describía la influencia del hombre en el medio, y la segunda, la influencia del medio en el hombre (Ortega, 2010, p. 126). Esa discusión se proyecta. Así, Ortega sostiene que la geografía política concibe al Estado como un ente estático, mientras que la geopolítica lo considera dinámico:

[...] la Geopolítica incluye el estudio del crecimiento, el cambio, la evolución y la dinámica de los espacios terrestres y de las fuerzas políticas que luchan entre ellos para alcanzar sus propios cometidos. La Geografía Política es eminentemente descriptiva del espacio del Estado, mientras la Geopolítica estudia y se plantea la cuestión dinámica del Estado. Se podría decir que la diferencia fundamental estriba en que la Geografía Política describe y expone. Por el contrario, la Geopolítica interpreta y predice los efectos que tendrán algunas variables geográficas sobre la constitución y desarrollo del Estado. (Ortega, 2010, p. 80)

Para Cheyre, el lugar de la geopolítica clásica es ocupado por la geoestrategia, que busca la convergencia entre la geografía militar y la estrategia. El desarrollo propio de la 
geoestrategia se encuentra en Nicholas Spykman, Richard Muir, Pierre Celerer, y Zbigniew Brzezinski, quienes se posicionan "en las áreas de las Relaciones Internacionales y Ciencias Militares" (Ortega, 2010, p. 10). Este planteamiento sigue a la escuela estadounidense, que no usa el término geopolítica por considerarlo incorrecto políticamente, y traza una división al menos terminológica entre la antigua y la nueva disciplina, en su afán por buscar situar la geopolítica en un contexto no conflictivo (Cheyre, 2010).

Finalmente, para Cheyre (1997), este estudio debe extenderse a países no limítrofes con el fin de generar "ejes de presión sobre otros Estados" (citado en Ortega, 2010, p. 13). Es indudable que el discurso de Cheyre sobre la disciplina busca cambiar la denominación y las connotaciones ligadas a su cultivo en el régimen militar, a la vez que lograr aprender la naturaleza cambiante del escenario internacional posterior a la Guerra Fría. Sobre la temática, describe Ortega (2010) lo siguiente:

La integración del territorio nacional; los ejes de desarrollo o corredores bioceánicos; la energía; el territorio antártico; las nuevas regiones del país (XIV y XV), son en definitiva algunas de las materias que permiten una mirada diferente de la situación privilegiada de Chile, por ello, se podría decir que la evolución del país ha estado precedida de decisiones políticas que han incluido una perspectiva geopolítica. (p. 133)

Para principios de los 2000, la geopolítica se diversifica. Si en los noventa se incorporaron planteamientos de la geopolítica crítica de Yves Lacoste, en los años siguientes se presenta una mezcla con la geoestrategia (Orueta, 1999). También aparecen geopolíticos en las universidades, que se fundamentan en autores principalmente europeos: Patricio Carvajal (2007), Carvajal y Monteverde (2012) y Juan Eduardo Mendoza (2018). En este contexto, el Centro de Estudios e Investigaciones Militares (CESIM) publicó Antología geopolitica de autores militares chilenos, cuyo editor fue Carlos Meirelles (2000). Para Jaime García Covarrubias, este es un libro fundamental:

Allí hay, entre otros, cinco trabajos del General Cañas, uno del Coronel Canessa (1985) sobre la regionalización, Coronel Von Chrismar y uno sobre el núcleo vital de Chile (pp. 236-252) tema hasta ese momento no tratado, de quien suscribe estas líneas. (respuesta a cuestionario, 8 de octubre de 2018)

En los 2000 se editó una antología de Cañas Montalva (2008) y se publicaron nuevos trabajos de Rodolfo Ortega $(2008 ; 2010 ; 2014)$, así como el último escrito de Von Chrismar (2010) antes de su muerte. Además de ello, se realizaron antologías militares con autores geopolíticos o se publicaron números especiales de Cuadernos de Difusión.

Hoy día, Ortega es el autor militar más prolífico y mantiene actualizados sus enfoques; por ejemplo, en 2009, incluyó a autores como Peter Taylor, Geografía política (2004) y John Agnew, Geopolitica, una revisión de la política mundial (2005). Ortega es un sintetizador de la vertiente clásica con las posturas renovadas, siempre conservando el dejo estatalista. Su obra más importante es Escenario y estrategia (Ortega, 2010). 
En el final de la fase transicional, empieza a destacarse la formación de académicos en las universidades, el diálogo con la geopolítica crítica, ya no solo la francesa, sino otras corrientes más modernas, y un nuevo ambiente para la geopolítica producto de los cambios del escenario global, especialmente debido al ascenso de China. Una peculiaridad es que la geopolítica de la Armada, más acotada, resulta de mayor vitalidad al vincularse con la proyección de la mayor estatura política estratégica del Estado de Chile, especialmente en el ámbito del Pacífico.

\section{Conclusiones}

Es indudable que la geopolítica se cultivó en Chile conforme muchas otras expresiones culturales y científicas de principios del siglo XX. En el contexto de su fundación, su temática fue tomada por la oficialidad chilena, que en ese momento era muy receptiva a los cambios de todo tipo. Así, su desarrollo permite perfilar una escuela geopolítica militar, trasmitida por la docencia y análisis militares, aunque no conformó planteamientos propios significativos. Su relevancia en algunos periodos de la historia nacional se debió a la confluencia entre los tomadores de decisiones políticos y los mandos militares en posición de consejeros. También fue más fuerte conforme el escenario vecinal presionaba con conflictos al norte y sur de Chile.

En la segunda etapa, su cultivo radicó en el circuito del Ejército y recibió una estandarización producto de dos autores: Cañas Montalva y Pinochet, pero sin solución de continuidad. En esta fase, la alineación se vuelve un factor fundamental para considerar el escenario mundial, si bien no de forma poderosa.

La tercera etapa coincide con el ascenso de Pinochet al poder y el traspaso de esa perspectiva a la toma de decisiones políticas. Se implementaron políticas específicas y, aunque el desarrollo teórico fue reducido, su aplicación fue relativamente amplia. Dada la autonomía militar, la geopolítica se institucionalizó en la Academia de Seguridad Nacional, antecesora de la Academia de Estudios Políticos y Estratégicos. El desarrollo de la geopolítica marítima es más amplio, menos destacado y se conecta con un escenario vecinal conflictivo.

La cuarta etapa, transicional, es el retorno al control civil. Allí la geopolítica se acomoda a los cambios políticos globales, toma nuevos temas, diversifica sus fuentes, trata de configurar un esquema más amplio en la perspectiva de asesorar al mando civil, pero hay otras perspectivas que ganan fuerza, como las políticas públicas. Al mismo tiempo, las hipótesis de conflicto ceden en vigencia, y la geopolítica se orienta hacia la integración y la cooperación. La geopolítica marítima se adapta al nuevo escenario y busca delimitar los intereses marítimos dentro de una conciencia acerca del valor del mar. Entretanto, el desarrollo geopolítico militar se empieza a reducir conforme asume un enfoque más institucional (Figura 1). 

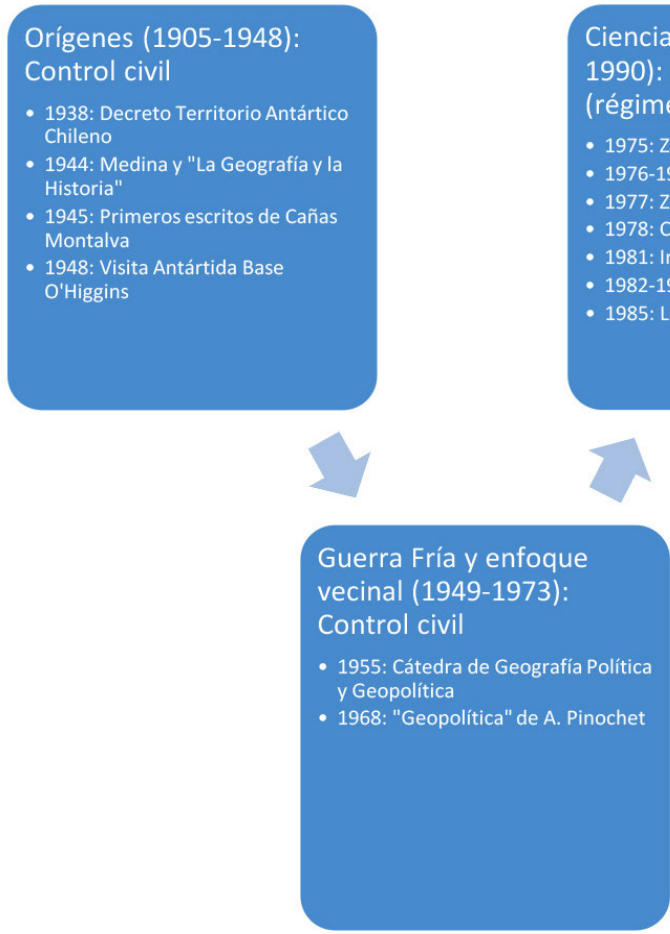

Ciencia del Estado (1973-

1990): Autonomía militar

(régimen militar)

- 1975: Zona franca de lquique

- 1976-1982: Carretera austral

- 1977: Zona franca de Punta Arenas

- 1978: Chile Geopolítico (Buzeta)

- 1981: Instituto Geopolítico de Chile

- 1982-1982: Carretera austral

- 1985: Ley Navarino

Figura 1. Contexto epocal de la geopolítica militar y marítima chilena.

Fuente: Elaboración propia.

En relación con sus orígenes, préstamos intelectuales y desarrollo, de la geopolítica chilena no se puede desprender una Escuela Geopolítica con mayúsculas, pero sí se puede hablar de un desarrollo conceptual que sigue una cierta lógica, por lo menos hasta la década de los 2000, cuando empieza a recibir enfoques críticos. Desde luego, su evolución está conectada con variables de contexto, representantes, temas, textos, naturaleza terrestre o marítima, su relación con el pensamiento civil y el escenario de control civil o autonomía militar. Ha sido más destacada en ciertas coyunturas, como el conflicto chileno-argentino, en buena parte del siglo XX; las administraciones del Partido Radical, muy cercanas a Cañas Montalva; o el golpe de 1973 y el ascenso de Pinochet que puso la geopolítica como preocupación. Tras los ańos noventa, cambió la coyuntura internacional, surgió la Globalización, se produjeron procesos intensos de integración entre Brasil y Argentina, se debilitó la percepción de Argentina como rival y, al mismo tiempo, hubo reajustes académicos en el enfoque geopolítico que los militares advierten y asimilan gradualmente. Es el momento de una geopolítica integracionista, y de volver a la preocupación por el poblamiento y la conexión de un país de miles de kilómetros y periferias despobladas. 
Así, en Chile, como en otras partes, el origen de la geopolítica estuvo unido indudablemente al desarrollo de la geografía: lo que la distingue es que su momento fundacional fue de primera mano (Kjellen) y se ha ido debilitando, mientras que la geografía ha reafirmado su estatuto científico. La geopolítica se plantea más como una disciplina. La geopolítica clásica reinó sin contrapeso hasta los noventa, cuando apareció un contexto internacional que cambió el eje del debate y provocó nuevas definiciones en su cultivo en el Ejército chileno. Por su parte, el desarrollo de una geopolítica marítima ha sido más lento, mientras que la Fuerza Aérea no ha ofrecido una reflexión geopolítica en sí.

\section{Declaración de divulgación}

El autor declara que no existe ningún potencial conflicto de interés relacionado con el artículo.

\section{Financiamiento}

El autor no declara fuente de financiamiento para la realización de este artículo.

\section{Sobre el autor}

Cristian Garay Vera es doctor en estudios americanos por la Universidad de Santiago de Chile y doctor en geografía e historia por la Universidad Nacional a Distancia de Madrid. Es profesor titular del Instituto de Estudios Avanzados de la Universidad de Santiago y director del Magíster en Política Exterior y de la Cátedra Manuel Bulnes. https://orcid.org/0000-0002-6575-7456 - Contacto: cristian.garay@usach.cl

\section{Referencias}

Amorós, M. (2019). Pinochet. Biografía militar y politica. Ediciones B.

Araya, J. (1997, 7 de septiembre). Geopolítica para el siglo XXI. El Mercurio, D26.

Arnello, M. (1985). Principios fundamentales para un proyecto nacional de Chile Futuro. Revista Geopolitica de Chile, 2.

Buzeta, Ó. (1978). Chile geopolitico. Presente y futuro. CESOC.

Cairo, H. (2011). La geopolítica como "ciencia del Estado": el mundo del general Haushofer. Geopolítica(s). Revista de Estudios sobre Espacio y Poder, 3(2), 337-345. https://bit.ly/3qPeFQH

Canessa R., J. (1982). Visión geopolítica de la regionalización chilena. Seguridad Nacional, 24, 13-36.

Cañas Montalva, R. (1959). Los mapas y la visión geopolítica del Pacífico. Revista Geográfica de Chile, 17.

Cañas Montalva, R. (2008). Geopolitica oceánica y austral. General Ramón Cañas Montalva [antología]. Academia de Guerra.

Carvajal Aravena, P. (2007). Geopolítica de los entornos y sociedad del riesgo. Una interpretación desde la geopolítica crítica: el caso chileno. Política y Estrategia, 108, 47-70. https://bit.ly/2SPE7Jl

Carvajal Aravena, P., \& Monteverde, A. (2012). La geopolitica maritima de los almirantes Buzeta, Ghisolfo y Martínez. Universidad de Playa Ancha. 
Caviedes, C. N. (1990). Aparición y desarrollo de doctrinas geopolíticas en los países del Cono Sur. En P. Kelly \& J. Child (Eds.), Geopolítica del Cono Sur y la Antártida. Editorial Pleamar.

Chateau, J. (1977). Características principales del pensamiento geopolítico chileno: análisis de dos libros. Flacso.

Cheyre, J. E. (1997). Inauguración de las I Jornadas Internacionales de Geopolítica. Memorial del Ejército de Chile, 456, 6-16.

Child, J., O’Tuathail, G., \& Dalby, S. (1998). Rethinking geopolitics. Routledge.

Comisión Nacional para el Estudio e Incorporación de las Fronteras Interiores al Desarrollo Nacional. (1994). Seminario Conquista y Consolidación de las Fronteras Interiores. Memorial, 445, 8-25.

"Confidencias". (1979, 4 de abril). Hoy, 97 (Santiago).

Devés, E., \& Álvarez, S. T. (2020). Problemáticas internacionales y mundiales desde el pensamiento latinoamericano. Ariadna Ediciones.

Di Giorgo, C. (1979). Chile, la geopolítica, y el mar. Revista de Marina, 5, 556-559.

Duvauchelle Rodríguez, M. (1996). La geopolítica y la oceanopolítica. Sus orígenes, fundamentos y relaciones; perspectiva chilena. Revista de Marina.

Gangas, M., \& Santis, H. (1987). La formación y el desarrollo de la geografía chilena. Revista de Geografía Norte Grande, 14, 76-79.

Garay Vera, C. (2006). En un entorno difícil: la existencia de la Academia de Guerra entre 1947 y 1970. En A. San Francisco (Ed.), La Academia de Guerra del Ejército de Chile 1886-2006 (pp. 143-170). Academia de Guerra; Bicentenario.

Garay Vera, C. (2008). Introducción. En R. Cañas Montalva, Geopolitica oceánica y austral. General Ramón Cañas Montalva (pp. 15-25). Academia de Guerra.

Garay Vera, C. (2017). La estrategia de la Guerra Fría. Política internacional y de defensa de Gabriel González Videla (Colección IDEA). Universidad de Santiago de Chile, Instituto de Estudios Avanzados.

García Arriagada, G. (1989). Visión geopolítica de la carretera longitudinal austral. Memorial del Ejército, 433, 90-122.

García Arriagada, G. (2005). ¿Quo Vadis Geopolítica? Memorial del Ejército, 476, 4-57.

García Covarrubias, J. (1984). Reflexiones sobre el núcleo vital de Chile. Memorial, 1/415, 73-85.

García Covarrubias, J. (1985). Reflexiones sobre el núcleo vital de Chile. Revista Geopolítica de Chile, 2(1).

Ghisolfo, F. (1979). Chile y el poder marítimo. "Mare est Vitale”. Politica y Estrategia, 13.

Ghisolfo, F. (1982). El poder naval frente al derecho del mar. Honorable Junta de Gobierno, Gabinete Armada.

Manzano, K. (2018). El sueño del libertador: O’Higgins y los indicios del pensamiento geopolítico chileno antártico. Revista Estudios Hemisféricos y Polares, 9(1), 26-38. https://bit.ly/3dNyeDB

Martínez Bush, J. (1993). Oceanopolitica: una alternativa para el desarrollo. Editorial Andrés Bello.

Medalla, E. (2008). La regionalización: reestructuración politico-administrativa bajo el Gobierno Militar (1973-1990) [tesis, Universidad de Valparaíso].

Meirelles, C. (Ed.). (2000), Antología geopolítica de autores militares chilenos. Centro de Estudios e Investigaciones Militares.

Mendoza, J. E. (2018). Razonamiento geopolitico. Construcción de representaciones y códigos geopolíticos de Chile y sus vecinos. Universidad de Concepción.

Ortega, R. (2008). Decisiones y desafíos geopolíticos de Chile. Cuadernos de Difusión, 11(28).

Ortega, R. (2009, julio-agosto). Decisiones y desafíos geopolíticos de Chile. Military Review, 61-70. https:// bit.ly/36eyuHD 
Ortega, R. (2010). Escenario y estrategia. Colección Academia de Guerra.

Ortega, R. (2014). Geopolítica: aporte del Ejército de Chile. Academia de Guerra.

Ortega, R. (2015). La geopolitica y geoestrategia chilena: ¿presente en el discurso y ausente en la práctica? (Cuaderno de Trabajo, 2). Centro de Estudios Estratégicos ANEPE CL. https://bit.ly/3hh37Cs

Orueta, P. (1999). Geoestrategia: conceptos y aplicaciones. Memorial del Ejército de Chile, 460.

Paulsen Bilbao, A. (2015). Los aportes de Friedrich Ratzel y Halford Mackinder en la construcción de la geografía política en tiempos de continuidades y cambios. Revista de Geografía Espacios, 5(9), 64-81. https://doi.org/10.25074/07197209.9.372

Peña, J. C. (2013). La secreta vida literaria de Augusto Pinochet. Debate.

Perey, C. (1986). Geopolítica Oceánica de Chile. Revista de Marina, 4.

Pinochet, A. (1953). Sintesis geográfica de Chile Argentina, Bolivia y Perú. Santiago de Chile.

Pinochet, A. (1967). Geografía militar. Interpretación militar de los factores geográficos. Santiago de Chile.

Pinochet, A. (1968). Geopolítica. Diferentes etapas para el estudio geopolítico de los Estados. Memorial del Ejército de Chile, 340-341.

Pinochet, A. (1984). Geopolitica. Editorial Andrés Bello (originalmente publicado en 1974).

Pinochet, A. (1990). Camino recorrido. Memorias de un soldado (vol. I). Instituto Geográfico Militar.

Pittman, H. T. (1990). De O'Higgins a Pinochet: Geopolítica aplicada en Chile. En P. Kelly \& J. Child (Eds.), Geopolitica del Cono Sur y la Antártida (pp. 177-187). Editorial Pleamar.

Riesco Jaramillo, R. (1985). Fronteras y tareas geopolíticas chilenas en el Océano Pacífico Sur y en el Continente Antártico. Revista Geopolitica de Chile, 2.

Rodríguez Tascón, G. (1950a). La geopolítica y sus teorías. Instituto Geográfico Militar.

Rodríguez Tascón, G. (1950b). La geopolítica y sus teorías. Memorial del Ejército de Chile, 9-10(222).

Rojas Sánchez, G. (2000). Chile escoge la libertad: la presidencia de Augusto Pinochet. Zig Zag.

Santelices, G. (1998). Estudio de la política de población y poblamiento en Chile. Política y Estrategia, 76.

Santis, H. (1988). El pensamiento geográfico-político de Ratzel en la geopolítica chilena. Revista de Geografía del Norte Grande, 25, 135-140.

Schiappacasse, M., Medalla, E., \& Sánchez, F. (2012). Allende y Pinochet. Las verdades olvidadas. Editorial Maye.

Strait, C. (1998). Conceptualización de las fronteras interiores. Aplicación a la XI Región del General Carlos Ibáñez del Campo. Política y Estrategia, 76.

Von Chrismar E., J. (1968). Leyes que se deducen del estudio de la expansión de los Estados. Biblioteca del Oficial.

Von Chrismar E., J. (2010). Los objetivos nacionales, base de la Politica nacional de los Estados. Academia de Guerra Santiago. 